\title{
Facial pain as first manifestation of anti-Hu paraneoplastic syndrome
}

\author{
Geneviève Demarquay • Adrien Didelot • Véronique Rogemond • \\ Philippe Ryvlin • Michel Gouttard • Paul Garassus • \\ François Mauguière $\cdot$ Jérome Honnorat
}

Received: 17 February 2010/Accepted: 1 April 2010/Published online: 13 April 2010

(C) Springer-Verlag 2010

\begin{abstract}
The diagnosis of anti-Hu-associated encephalomyelitis/sensory neuropathy may be particularly difficult when cranial nerve involvement represents the first clinical manifestation of the disease. We report a case of a patient who presented with facial pain as the first manifestation of an anti-Hu paraneoplastic syndrome, which needs a rapid detection and treatment of the underlying tumour. We suggest that paraneoplastic neuropathy should be considered during the management of trigeminal neuropathic pain, especially when brain imagery is normal.
\end{abstract}

Keywords Paraneoplastic syndrome .

Trigeminal neuropathy $\cdot$ Anti-Hu $\cdot$ Cancer $\cdot$ Facial pain

G. Demarquay $(\square)$

Service de Neurologie, Hôpital de la Croix-Rousse, 103, Grande Rue de la Croix-Rousse, 69004 Lyon, France e-mail: genevieve.demarquay@chu-lyon.fr

G. Demarquay · A. Didelot · V. Rogemond · J. Honnorat Centre de Référence des Syndromes Paranéoplasiques, Neurological Hospital, Lyon, France

P. Ryvlin · F. Mauguière

Department of Neurology, Neurological Hospital,

Lyon, France

M. Gouttard

Department of Neurology, Bourg en Bresse Hospital,

Bourg en Bresse, France

P. Garassus

Department of Neurology, Clinique du Tonkin,

Villeurbanne, France

\section{Introduction}

Management of facial pain can be difficult regarding aetiology diagnosis. Trigeminal neuropathic pain requires specialised investigations to exclude a structural lesion involving the fifth cranial nerve in its full course, from its nuclei in the brainstem to its peripheral branches. If this investigation is normal, screening laboratory tests must be considered to exclude a neuropathy caused by infectious agents or connective tissue diseases and vasculitis. Rare causes of sensory neuropathy may also be considered such as paraneoplastic neuropathy, which needs a rapid detection and treatment of the tumour. We report a case of a patient who presented with trigeminal neuropathic pain as the first manifestation of an anti-Hu-associated brainstem encephalitis.

\section{Case report}

A non smoker 74-year-old woman presented with left facial pain of 2-month duration. She complained from an intermittent burning sensation located in the second division of the left trigeminal nerve (V2) and reported cheek numbness. Sensory stimulation of the face did not precipitate facial pain. The patient also reported a 1-month history of asthenia and diarrhoea. The first patient's general examination disclosed normal findings including normal facial sensitivity. There was no palpable regional lymph node. The oro-facial examination was normal. No abnormality was detectable on repeated brain MRI with gadolinium.

After 3 months, the patient reported dry mouth and dry eyes confirmed by a Shirmer's test. Neurological examination revealed hypoesthesia in the left maxillary branch. 
Blink reflex was normal. Electromyography and nerve conduction tests were not conducted because the patient had no clinical symptom of peripheral neuropathy. Clonazepam and pregabaline treatments were administrated without pain relief. The brain MRI remained normal. Blood tests showed elevated erythrocyte sedimentation rate (ESR) (44 for a normal $<20$ ) but normal C-reactive protein. Glycosylated hemoglobin and liver function tests were normal. Immunologic tests showed negative anti-Ro/ anti-SS-A, anti-SS-B antibodies, antinuclear antibody, anti-neutrophil cytoplasmic antibodies (ANCAs). Serum angiotensin-converting enzyme was normal. Temporal artery biopsy did not reveal giant cell arteritis. Labial salivary gland biopsy for Sjögren's syndrome was negative. A broad serological screening for infectious conditions including HIV, herpes simplex virus 1 and 2, hepatitis, Lyme and syphilis was negative. The cerebrospinal fluid (CSF) analysis showed oligoclonal bands with normal protein levels. No malignant cells were disclosed. Anti-Hu antibodies were detected in the serum with dot blot and indirect immunofluorescence techniques [1].

To localise the suspected malignancy, the patient underwent computed tomography (CT) scans of the chest and abdomen which disclosed an axillary lymph node (Fig. 1) and retroperitoneal lymph nodes. The results of the mammography, gastroscopy, coloscopy, bone marrow biopsy were normal. The whole body PET/CT scan demonstrated increased uptake of 18-F-FDG in the axillary and retroperitoneal lymph nodes, but disclosed no primitive tumour. The biopsy of the axillary lymph node diagnosed a small cell lung carcinoma. We concluded to the diagnosis of anti-Hu trigeminal neuropathy as a first manifestation of paraneoplastic brainstem encephalitis and suggested that the diarrhoea, dry month and dry eyes were due to paraneoplastic dysautonomia. A chemotherapy treatment with a combination of cisplatin and etoposide was administrated. The outcome was rapidly unfavourable. After 2 months, the patient developed dysarthria and dysphagia. The chest CT scan showed an increased size of the axillary lymph node. A second-line chemotherapy was introduced without success. The patient died 14 months after the first signs of trigeminal numbness.

\section{Discussion}

Trigeminal nerve involvement is a frequent cause of facial pain. In our patient, cheek numbness and further trigeminal hypoesthesia strongly suggested symptomatic trigeminal neuropathy. A structural lesion involving the fifth cranial nerve was excluded by the normality of the brain MRI. Vasculitis and connective tissue diseases associated with trigeminal neuropathy were then considered. The

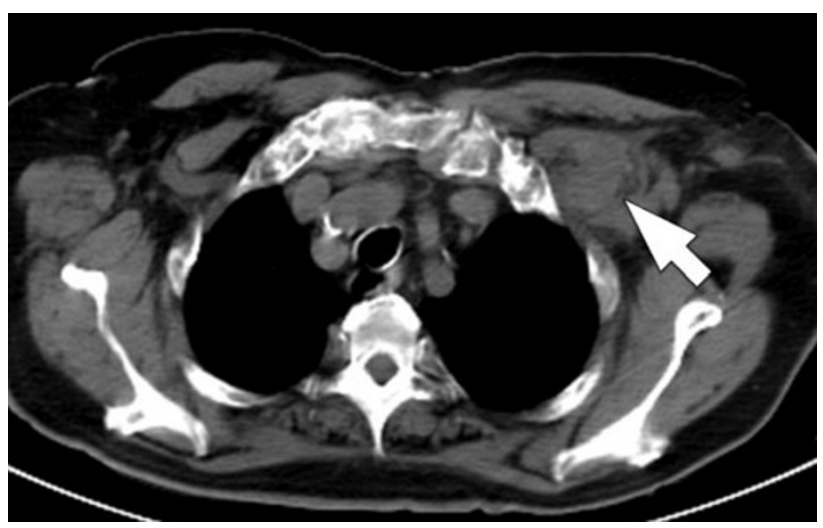

Fig. 1 Left axillary lymph node revealed by the chest CT scan

association of xerophthalmia and xerostomia suggested a Sjögren's syndrome, which is associated with a sensory trigeminal involvement in 16\% [2]. However, the absence of anti-Ro/anti-SS-A, anti-SS-B antibodies and the normality of the labial salivary gland biopsy argued against this diagnosis. Trigeminal neuropathy has been also reported in association with other connective tissue diseases, such as scleroderma or mixed connective tissue diseases [3], but the lack of joint, renal, eye, skin involvement, and the normality of the immunologic tests prompted us to reject these diagnoses. There was no evidence of infection on serological screening.

Facial pain as a first manifestation of a cancer was also considered. Trigeminal neuropathic pain can be associated with cancer, either by a direct compression of the nuclei in the brainstem or of the peripheral branches by the tumour or by a meningeal carcinomatosis. In our patient, we did not disclose any evidence of metastatic invasion nor contiguity spread of a tumour lesion to the trigeminal nerve. Conversely, the CSF oligoclonal bands and the detection of anti-Hu antibody confirmed the hypothesis of a paraneoplastic syndrome.

Cranial nerve involvement represents one of the manifestations of anti-Hu-associated brainstem encephalitis [4] which is associated with a small-cell lung carcinoma in the majority of patients. Brainstem symptoms can be isolated or associated during paraneoplastic encephalomyelitis with signs of widespread dysfunction of the nervous system [1, $5,6]$. The frequency of trigeminal involvement in patients with anti-Hu antibodies is unknown. Facial numbness was described in 2 out of 23 patients with brainstem encephalitis reported by Dalmau et al [6]. Trigeminal involvement can emerged early in the course of paraneoplastic syndrome with anti-Hu antibodies in association with multiple cranial nerve palsies [7] or with progressive dysautonomia [8]. To our knowledge, only one previous study has reported trigeminal involvement as the first manifestation of an anti-Hu neuropathy [9]. However, we suggest that 
trigeminal paraneoplastic involvement may be underestimated. 33 cases of facial pain as the first manifestation of lung cancers have been collected in literature in 2003 [10]. In these patients, the mean delay between the onset of facial pain and the diagnosis of lung cancer was 9 months. In most cases, the pain was described as severe and aching and commonly affected the jaws and temporal region. In two patients, the diagnosis of trigeminal neuralgia was previously made. Several mechanisms were proposed to explain facial pain, such as local invasion, compression of the nerve or a paraneoplastic syndrome. To our knowledge, screening for paraneoplastic antibodies was not performed in these studies.

In conclusion, trigeminal neuropathy is a well-known symptom occurring during the evolution of patients with anti-Hu-associated encephalomyelitis/sensory neuropathy, but can also represent the first isolated manifestation of a paraneoplastic syndrome.

Conflict of interest None.

\section{References}

1. Honnorat J, Cartalat-Carel S, Ricard D et al (2009) Onco-neural antibodies and tumor type determine survival and neurological symptoms in paraneoplastic neurological syndromes with $\mathrm{Hu}$ or
CV2/CRMP5 antibodies. J Neurol Neurosurg Psychiatry 80:412416

2. Mori K, Iijima M, Koike $\mathrm{H}$ et al (2005) The wide spectrum of clinical manifestations in Sjögren's syndrome-associated neuropathy. Brain 128:2518-2534

3. Peñarrocha M, Cervelló MA, Martí E, Bagán JV (2007) Trigeminal neuropathy. Oral Dis 13:141-150

4. Saiz A, Bruna J, Stourac P et al (2009) Anti-Hu-associated brainstem encephalitis. J Neurol Neurosurg Psychiatry 80:404407

5. Dalmau J, Graus F, Rosenblum MK, Posner JB (1992) Anti-Huassociated paraneoplastic encephalomyelitis/sensory neuronopathy: a clinical study of 71 patients. Medicine 71:59-72

6. Graus F, Keime-Guibert F et al (2001) Anti-Hu-associated paraneoplastic encephalomyelitis: analysis of 200 patients. Brain 124:1138-1148

7. Fujimoto S, Kumamoto T, Ito T et al (2002) A clinicopathological study of a patient with anti-Hu-associated paraneoplastic sensory neuronopathy with multiple cranial nerve palsies. Clin Neurol Neurosurg 104:98-102

8. Winkler AS, Dean A, Hu M, Gregson N, Chaudhuri KR (2001) Phenotypic and neuropathologic heterogeneity of anti-Hu antibody-related paraneoplastic syndrome presenting with progressive dysautonomia: report of two cases. Clin Auton Res $11: 115-118$

9. Raaphorst J, Vanneste J (2006) Numb cheek syndrome as the first manifestation of anti-Hu paraneoplastic neuropathy. J Neurol 253:664-665

10. Sarlani E, Schwartz AH, Greenspan JD, Grace EG (2003) Facial pain as first manifestation of lung cancer: a case of lung cancerrelated cluster headache and a review of the literature. J Orofac Pain 17:262-267 